\title{
Our Brains are Targets No. 1, Cleared for Firing - an Overview
}

\author{
Doepp Manfred* \\ Holistic Center 13 Haupt. St., Abtwil 9030, Switzerland \\ *Corresponding author: Manfred D, Holistic Center 13 Haupt. St., Abtwil 9030, Switzerland. \\ To Cite This Article: Doepp Manfred. Our Brains are Targets No. 1, Cleared for Firing - an Overview. Am J Biomed Sci \& Res. 2021 - 14(3). AJBSR. \\ MS.ID.001986. DOI: 10.34297/AJBSR.2021.14.001986.
}

Received: 眥August 09, 2021; Published: 眥 September 30, 2021

\section{Opinion}

The number of brain diseases is constantly increasing. There are a multitude of causes for this. Our organism has a nature-electric structure, it works analogously and is a part of nature. However the environment as well as the inner world are becoming more and more digital and thus unnatural. The entry points for the brain are naturally the sense organs who are suffering.

a) the eyes: flickering light, neon tubes, LEDs, blue light from monitors, no more black nights

b) the ears: no more silence, cacophony, constant noise, rock and pop

c) the endocrine glands of the brain: technical electrosmog (new: 5G), Haarp, geoengineering, fluorides, light metals, chemtrails, Schumann frequency changes

The infestation with neurotropic viruses is increasingly developing into widespread diseases: EBV, cytomegaloviruses, herpes viruses, spike proteins; also other pathogens: Borrelia, Toxoplasma... While the blood-brain barrier used to provide a shield, this is now increasingly perforated. Spike proteins from mRNA vaccinations produce brain venous thrombosis.

Unfortunately, dentistry is another issue. While dentists used to prefer a revealing therapy, i.e. pulling out diseased teeth, a covering therapy has developed in recent decades. The aim is to preserve diseased teeth, e.g. with root canal treatments. The more academic dentistry became, the more the relationship between foreign substances in the mouth and the health of the whole body was ignored.
What does complementary medicine say about this? It says that dentists should see their primary task as making the mouth toxin-free, not maintaining or even producing foci/toxins, but removing anything that might be toxic from the mouth. What are the poisons that come into consideration? They are: fluorides, mercury, palladium, platinum (e.g. in dental gold) and other heavy metals, but also dead tissue = cadaveric toxins, and above all foci in the sense of inflammations, granulomas and foci of all kinds.

In more recent times, efforts to achieve a superficially aesthetic dental appearance have led to implants taking the place of previously common prosthetic restorations. In principle, there is nothing negative about this, as long as ceramic is used, which is usually tolerated by the body. However, titanium pins are used today, which (in line with their use in artificial joint prostheses) do not seem to cause allergies or rejection when they first come into contact with the organism.

More recently, however, bioresonance therapists have noticed that titanium is less and less tolerated after a few months. This runs parallel to the exposure to chemtrails, which contain titanium as an important component, and to the titanium oxide admixtures in many medicines. Whereas heavy metals used to be the main problem, today light metals have been added, most clearly visible in the aluminium poisoning of the brain and especially our pineal glands.

\section{New Topics, NICO and RANTES}

Now to the RANTES. It is thanks to colleagues Lechner and von Baehr that they have uncovered the relationship between chronic 
osteitis of the jaw of the NICO type (non-infectious chronic osteitis) and serious diseases in the body. This concerns mainly cancer, in women preferentially breast cancer. Regarding NICO, there are several problems: a) it hardly causes any pain or other subjective discomfort, b) it is not known/recognised by most dentists, c) it is difficult to detect in normal x-rays.

For decades, the author sent patients who showed dental focus findings in the bioresonance to the dentist with the request of a focus clarification. Most of them came back with the result "everything is fine, there is nothing there". When computer tomography came along with the possibility of assessing the jaw bones more precisely, patients surprisingly came back after the CT with the result of a tooth root or jaw bone focus. The time of objectifying the suspicious findings began.

Now one could object that this is, so to speak, dental esotericism. However, this objection is no longer valid since the objective blood value of the RANTES became analysable in such cases. RANTES is a chemokine that is detectable in elevated levels in NICO and represents, among other things, oxidative stress in NICO (https:// de.wikipedia.org/wiki/Chemokin).

\section{Quote from a Publication by Lechner and Von Baehr}

(https://www.researchgate.net/publication/319057541_ Kieferherd_und_systemische_Entzundungen_-_Literaturrecherche_ zu_RANTES_Publikationen), as well as: https://www.dr-lechner. de/kieferostitis/

"For decades, phenomena such as "jaw ostitis" and "NICO" have been causally linked to general diseases as so-called "foci". Energy pathways of Chinese acupuncture have also served as holistic functional and explanatory models. The authors' research shows with modern laboratory methods that over-activated signal transduction cascades, especially of the chemokine RANTES in osteolytic changes of the jaw bone, can be linked to RANTES effects in complex chronic diseases. We thus present an extended explanatory model of the so-called "interference field effect" from the tooth-jaw area and thus promote complementary and integrative thinking in medicine and dentistry. The modern increase in knowledge about the pathophysiological role of the chemokine RANTES, however, contrasts with minimal hits in relation to osteopathies of the jaw bone in a corresponding literature search. This extreme imbalance is presented and critically questioned."

\section{Example}

A patient with breast cancer came for a Time Waver ${ }^{\circledR}$ examination every 2 months. She rejected orthodox medicine. Each time, a NICO suspicion came up, among other things, concerning the area $37 / 38$. She went to her family dentist, who found nothing in the normal panoramic X-ray. Then she chose a complementary dentist who was able to confirm the findings on CT. He sent blood for RANTES determination to a laboratory, with the result of an increase to double. She wrote in her email of 05.07.2021: "We will definitely continue with the amalgam removal and he also recommended that I visit the dentist Dr. J. L. in Munich, who can do a CaviTAU ultrasound examination to determine possible diseases of the jaw. RANTES is one of these diseases. The RANTES value is 58.2 and should be less than 30. I think I have found the cause of the breast cancer. RANTES is responsible for many diseases, including breast cancer. You had always seen during the bioresonance therapy that I had an inflammation in my jaw."

This case is exemplary for the topic of NICO and RANTES. May this knowledge spread as widely as possible. The so frequent separation of dentistry and holistic medicine must not be maintained.

\section{Oral Cavity, Lymph flow and Brain}

A general issue today is the insufficient lymphatic drainage of the head and thus the brain. This is accompanied by "silent inflammation" of the glial tissue, a special type of mitochondriopathy in the brain, along with anti-RNA antibodies. The insufficient brain detoxification leads to an oversaturation of free radicals or oxidative stress. Unfortunately, the oral cavity is an important source of toxins and thus an overload of the lymphatic drainage of the head. It is not only a matter of organic disorders, but of disorders of consciousness, of loss of consciousness, of vegetation instead of an individual life.

The brain suffers, it develops diseases. The eyes and the ENT organs are also affected. To get the lymph flow moving, head and neck lymph drainage is useful, but insufficient. Vibration therapy of the head and neck, especially with the connective tissue frequency of approx. $25 \mathrm{~Hz}$ according to Vladimir Nazarov (Biomechanical Stimulation = BMS) https://www.altmeyers.org/de/dermatologie/ biomechanische-stimulation-14032. Another possibility of an autotherapy of the brain is the long-lasting humming of "OM". Additionally it promotes the state of our consciousness.

\section{Summary}

Our brains are targets nowadays of different origines like it has never been before. We have to know about that in order to protect ourselves, and to protect our most precious organ. 